PROCEEDINGS OF THE

AMERICAN MATHEMATICAL SOCIETY

Volume 136, Number 7, July 2008, Pages 2483-2492

S 0002-9939(08)09384-2

Article electronically published on March 7, 2008

\title{
WEIGHTED REPRODUCING KERNELS AND TOEPLITZ OPERATORS ON HARMONIC BERGMAN SPACES ON THE REAL BALL
}

\author{
RENATA OTÁHALOVÁ
}

(Communicated by Michael T. Lacey)

\begin{abstract}
For the standard weighted Bergman spaces on the complex unit ball, the Berezin transform of a bounded continuous function tends to this function pointwise as the weight parameter tends to infinity. We show that this remains valid also in the context of harmonic Bergman spaces on the real unit ball of any dimension. This generalizes the recent result of C. Liu for the unit disc, as well as the original assertion concerning the holomorphic case. Along the way, we also obtain a formula for the corresponding weighted harmonic Bergman kernels.
\end{abstract}

\section{INTRODUCTION}

Let $\mathbb{B}^{n}$ be the ball in $\mathbb{R}^{n}, n \geq 2$, and $d z$ the Lebesgue measure on $\mathbb{B}^{n}$. For $\alpha>-1$, consider the measure

$$
d A_{\alpha}(z):=c_{\alpha}\left(1-|z|^{2}\right)^{\alpha} d z
$$

where

$$
c_{\alpha}=\frac{\Gamma\left(\alpha+\frac{n}{2}+1\right)}{\pi^{\frac{n}{2}} \Gamma(\alpha+1)}
$$

is chosen so as to make $d A_{\alpha}$ a probability measure. For simplicity, we will usually assume that $\alpha$ is an integer.

The harmonic Bergman space $L_{\text {harm }}^{2}\left(\mathbb{B}^{n}, d A_{\alpha}\right)$ consists, by definition, of all harmonic functions in $L^{2}\left(\mathbb{B}^{n}, d A_{\alpha}\right)$. It is known that point evaluation functionals are continuous on the harmonic Bergman space, so it possesses a reproducing kernel; i.e., there exists a function $R_{\alpha}(x, y)$ on $\mathbb{B}^{n} \times \mathbb{B}^{n}$, harmonic in each variable, such that

$$
f(x)=\int_{\mathbb{B}^{n}} f(y) R_{\alpha}(x, y) d A_{\alpha}(y)
$$

for each $f \in L_{\text {harm }}^{2}\left(\mathbb{B}^{n}, d A_{\alpha}\right)$ and for each $x \in \mathbb{B}^{n}$.

Received by the editors March 5, 2007.

2000 Mathematics Subject Classification. Primary 47B35; Secondary 32A25, 31B05, 33C55.

Key words and phrases. Reproducing kernel, Toeplitz operators, harmonic Bergman space.

This research was supported by projects 201/03/H152 from the Grant Agency of the Czech Republic, and MSM 4781305904 from the Czech Ministry of Education.

(C)2008 American Mathematical Society Reverts to public domain 28 years from publication 
The Berezin transform of a bounded linear operator $T$ on $L_{\text {harm }}^{2}\left(\mathbb{B}^{n}, d A_{\alpha}\right)$ is the function $\tilde{T}^{\alpha}(z)$ on $\mathbb{B}^{n}$ defined by

$$
\tilde{T}^{(\alpha)}(z)=\frac{\left\langle T^{(\alpha)} R_{\alpha z}, R_{\alpha z}\right\rangle}{\left\langle R_{\alpha z}, R_{\alpha z}\right\rangle}=\frac{T^{(\alpha)} R_{\alpha z}(z)}{R_{\alpha}(z, z)},
$$

where, for the sake of brevity, we have denoted $R_{\alpha z}(w):=R_{\alpha}(z, w)$.

Finally, for $f \in L^{\infty}\left(\mathbb{B}^{n}\right)$, the Toeplitz operator $T_{f}$ with symbol $f$ is the operator on $L_{\text {harm }}^{2}\left(\mathbb{B}^{n}, d A_{\alpha}\right)$ defined by

$$
T_{f} g=Q_{\alpha}(f g),
$$

where $Q_{\alpha}: L_{\text {harm }}^{2}\left(\mathbb{B}^{n}, d A_{\alpha}\right) \rightarrow L_{\text {harm }}^{2}\left(\mathbb{B}^{n}, d A_{\alpha}\right)$ is the orthogonal projection. That is,

$$
T_{f} g(z)=\int_{\mathbb{B}^{n}} g(x) f(x) R_{\alpha}(z, x) d A_{\alpha}(x) .
$$

It was shown by C. Liu [7] that if $n=2$ (so that $\mathbb{B}^{2}$ is just the unit disc in the complex plane $\mathbb{C})$, then for $f \in C\left(\overline{\mathbb{B}^{n}}\right)$,

$$
\begin{aligned}
\tilde{T}_{f}^{(\alpha)} & \rightarrow f & \text { uniformly, and } \\
\left\|T_{f}^{(\alpha)}\right\| & \rightarrow\|f\|_{\infty} &
\end{aligned}
$$

as $\alpha \rightarrow \infty$.

This extends the same result known previously for Toeplitz operators on Bergman spaces of holomorphic functions, which finds important applications in mathematical physics (quantization on Kähler manifolds; see e.g. [4).

The aim of the present paper is to generalize Liu's result also to $n \geq 3$. To do this, we first establish a (reasonably) explicit formula for the kernels $R_{\alpha}(x, y)$; this is done in Section 2. Our main result (the generalization of (11) and (2) ) is proved in Section 3 ,

We remark that we actually obtain a somewhat stronger result than (11); namely, we show that for any $f \in B C\left(\mathbb{B}^{n}\right):=C\left(\mathbb{B}^{n}\right) \cap L^{\infty}\left(\mathbb{B}^{n}\right)$ we also have

$$
\tilde{T}_{f}^{(\alpha)}(z) \rightarrow f(z)
$$

as $\alpha \rightarrow \infty$ for all $z \in \mathbb{B}^{n}$. This gives a new piece of information even for the original case $n=2$.

The author thanks the referee for very helpful comments.

\section{ReProducing KERNEL}

In this part we will find an explicit formula for the reproducing kernel of the space $L_{\text {harm }}^{2}\left(\mathbb{B}^{n}, d A_{\alpha}\right)$ of all harmonic functions on the unit ball in $\mathbb{R}^{n}$ square-integrable with respect to the measure $d A_{\alpha}(y)$, when $\alpha$ is an integer. Let $\mathcal{H}_{m}\left(\mathbb{R}^{n}\right)$ denote the space of the harmonic polynomials on $\mathbb{R}^{n}$ that are homogeneous of degree $m$. For each $x \in \mathbb{R}$, there exists a unique function $\mathcal{Z}_{m}(\cdot, x) \in \mathcal{H}_{m}(S)$ such that

$$
p(x)=\int_{S} p(\xi) \overline{\mathcal{Z}_{m}(\xi, x)} d \sigma(\xi),
$$

for all $p \in \mathcal{H}_{m}(S)$. The polynomial $\mathcal{Z}_{m}(\cdot, x)$ is called the zonal harmonic of degree $m$ and pole $x$. See e.g. [3. p. 94]. It extends to a function on $\mathbb{R}^{n} \times \mathbb{R}^{n}$ by setting

$$
\mathcal{Z}_{m}(x, y)=|x|^{m}|y|^{m} \mathcal{Z}_{m}(x /|x|, y /|y|)
$$

for $m>0$, and for $m=0, \mathcal{Z}_{0}=1$. 
Passing to polar coordinates $z=r \xi(r>0, \xi \in S)$, the Lebesgue measure becomes

$$
d z=\frac{2 \pi^{n / 2}}{\Gamma\left(\frac{n}{2}\right)} r^{n-1} d r d \sigma(\xi) .
$$

From (3), we have therefore for any $p \in \mathcal{H}_{m}$,

$$
\begin{aligned}
& \int_{\mathbb{B}^{n}} p(y) \mathcal{Z}_{m}(x, y) d A_{\alpha}(y) \\
& =\frac{2 \Gamma\left(\frac{n}{2}+\alpha+1\right)}{\alpha ! \Gamma\left(\frac{n}{2}\right)} \int_{0}^{1} r^{n-1}\left(1-r^{2}\right)^{\alpha} \int_{S} p(r \xi) \mathcal{Z}_{m}(x, r \xi) d \sigma(\xi) d r \\
& =\frac{2 \Gamma\left(\frac{n}{2}+\alpha+1\right)}{\alpha ! \Gamma\left(\frac{n}{2}\right)} \int_{0}^{1} r^{n+2 m-1}\left(1-r^{2}\right)^{\alpha}\left(\int_{S} p(\xi) \mathcal{Z}_{m}(x, \xi) d \sigma(\xi)\right) d r \\
& =\frac{2 \Gamma\left(\frac{n}{2}+\alpha+1\right)}{\alpha ! \Gamma\left(\frac{n}{2}\right)} p(x) \int_{0}^{1} r^{n+2 m-1}\left(1-r^{2}\right)^{\alpha} d r \\
& =\frac{\Gamma\left(\frac{n}{2}+\alpha+1\right) \alpha !\left(m+\frac{n}{2}-1\right) !}{\alpha ! \Gamma\left(\frac{n}{2}\right)\left(\alpha+m+\frac{n}{2}\right) !} p(x)
\end{aligned}
$$

for each $x \in \mathbb{R}^{n}$.

Now recall that for any orthonormal basis $\left\{\varphi_{j}\right\}$ of $L_{\text {harm }}^{2}\left(\mathbb{B}^{n}, d A_{\alpha}\right)$, the reproducing kernel $R_{\alpha}$ is given by

$$
R_{\alpha}(x, \bar{y})=\sum_{j=1}^{\infty} \varphi_{j}(x) \overline{\varphi_{j}(y)} .
$$

(See e.g. 2].) Now each $\mathcal{H}_{m}$ is a closed subspace of $L_{\text {harm }}^{2}\left(\mathbb{B}^{n}, d A_{\alpha}\right)$, the spaces $\mathcal{H}_{m}$ and $\mathcal{H}_{k}$ are orthogonal if $m \neq k$, and the span of all $\mathcal{H}_{m}, m \geq 0$, is the whole $L_{\text {harm }}^{2}\left(\mathbb{B}^{n}, d A_{\alpha}\right)$; in other words,

$$
\bigoplus_{m=1}^{\infty} \mathcal{H}_{m}=L_{\text {harm }}^{2}\left(\mathbb{B}^{n}, d A_{\alpha}\right) .
$$

Thus if we choose a basis $\left\{\varphi_{m j}\right\}_{j=1}^{\operatorname{dim} \mathcal{H}_{m}}$ in each $\mathcal{H}_{m}$ so that

$$
\sum_{j=1}^{\operatorname{dim} \mathcal{H}_{m}} \varphi_{j}(x) \overline{\varphi_{j}(y)}:=K_{m}(x, y)
$$

is the reproducing kernel of $\mathcal{H}_{m}$, then $\bigcup_{m=0}^{\infty}\left\{\varphi_{m j}\right\}_{j=1}^{\operatorname{dim} \mathcal{H}_{m}}$ is a basis for the whole $L_{\text {harm }}^{2}\left(\mathbb{B}^{n}, d A_{\alpha}\right)$; consequently,

$$
K(x, y)=\sum_{m}^{\infty} K_{m}(x, y) .
$$

On the other hand, from (4) we get

$$
K_{m}(x, y)=\frac{\Gamma\left(\frac{n}{2}\right)\left(\alpha+m+\frac{n}{2}\right) !}{\Gamma\left(\frac{n}{2}+\alpha+1\right)\left(m+\frac{n}{2}-1\right) !} \mathcal{Z}_{m}(x, y) .
$$

Thus we arrive at the following result. 


\section{Proposition 2.1.}

$$
R_{\alpha}(x, y)=\sum_{m=0}^{\infty} \frac{\Gamma\left(\frac{n}{2}\right)\left(\alpha+m+\frac{n}{2}\right) !}{\Gamma\left(\frac{n}{2}+\alpha+1\right)\left(m+\frac{n}{2}-1\right) !} \mathcal{Z}_{m}(x, y) .
$$

It should be noted that this result is definitely not new, see e.g. [5. p. 32 (1)], or [6. Section 2], or [9, Proposition 3], but it is convenient to recall it here.

For $\alpha$ an integer, the last sum can be summed explicitly. Recall [3, p. 178] that the usual Poisson kernel

$$
P(x, y)=\frac{1-|x|^{2}|y|^{2}}{\left(1-2 x \cdot y+|x|^{2}|y|^{2}\right)^{\frac{n}{2}}}
$$

is equal to

$$
P(x, y)=\sum_{m=0}^{\infty} \mathcal{Z}_{m}(x, y),
$$

for $x, y \in B$. Hence, for $\alpha$ an integer $(\alpha=0,1,2, \ldots)$ we have

$$
\begin{aligned}
\left(t \frac{d}{d t}+\alpha\right) P(t x, y) & =\sum_{m=0}^{\infty}\left(t \frac{d}{d t}+\alpha\right) \mathcal{Z}_{m}(t x, y) \\
& =\sum_{m=0}^{\infty}\left(t \frac{d}{d t}+\alpha\right) t^{m} \mathcal{Z}_{m}(x, y) \\
& =\sum_{m=0}^{\infty}(m+\alpha) t^{m} \mathcal{Z}_{m}(x, y)
\end{aligned}
$$

By iteration it follows that

$$
\begin{aligned}
\left(t \frac{d}{d t}+\frac{n}{2}\right) \ldots\left(t \frac{d}{d t}+\right. & \left.\frac{n}{2}+\alpha\right) P(t x, y) \\
& =\sum_{m=0}^{\infty}\left(m+\frac{n}{2}\right) \quad \ldots \quad\left(m+\frac{n}{2}+\alpha\right) t^{m} \mathcal{Z}_{m}(x, y) .
\end{aligned}
$$

Consequently,

$$
\begin{aligned}
\left(t \frac{d}{d t}+\frac{n}{2}\right) & \left.\ldots\left(t \frac{d}{d t}+\frac{n}{2}+\alpha\right) P(t x, y)\right|_{t=1} \\
& =\sum_{m=0}^{\infty}\left(m+\frac{n}{2}\right) \ldots\left(m+\frac{n}{2}+\alpha\right) t^{m} \mathcal{Z}_{m}(x, y) \\
& =\sum_{m=0}^{\infty} \frac{\left(m+\frac{n}{2}+\alpha\right) !}{\left(m+\frac{n}{2}-1\right) !} \mathcal{Z}_{m}(x, y) \\
& =\frac{2 \Gamma\left(\frac{n}{2}+\alpha+1\right)}{\alpha ! \Gamma\left(\frac{n}{2}\right)} \alpha ! R_{\alpha}(x, y)
\end{aligned}
$$

so we get the following formula.

Proposition 2.2. Let $x, y \in B$. Then

$$
R_{\alpha}(x, y)=\left.\frac{\Gamma\left(\frac{n}{2}\right)}{2 \Gamma\left(\frac{n}{2}+\alpha+1\right)} \mathcal{D}_{\alpha+1} P(t x, y)\right|_{t=1},
$$


where

$$
\mathcal{D}_{\alpha+1}=\left(t \frac{d}{d t}+\frac{n}{2}\right)\left(t \frac{d}{d t}+\frac{n}{2}+1\right) \ldots\left(t \frac{d}{d t}+\frac{n}{2}+\alpha\right) .
$$

Remark. The formula (6) has apparently also appeared in [5, p. 32 (1)], though in the somewhat different form

$$
R_{\alpha}(x, y)=\left[\rho^{1-n}\left(\frac{\partial}{\partial \rho}\right)^{\alpha+1} \rho^{n+\alpha} P\left(x, \rho^{2} y^{\prime}\right)\right]_{\rho=\sqrt{|y|}} .
$$

\section{Main ReSUlt}

Recall that the Möbius transformation $\varphi_{z}$ is the smooth map of $\mathbb{B}^{n}$ onto itself defined for each $z \in \mathbb{B}^{n}$ by

$$
\varphi_{z}(w)=\frac{|w-z|^{2} z-\left(1-|z|^{2}\right)(w-z)}{1-2\langle w, z\rangle+|w|^{2}|z|^{2}},
$$

where $\langle w, z\rangle:=w_{1} z_{1}+\cdots+w_{n} z_{n}$ denotes the usual scalar product in $\mathbb{R}^{n}$. In the next lemma, we summarize the properties of the mapping $\varphi_{z}$. The proofs can be found e.g. in [1].

Lemma 3.1. For every $z \in \mathbb{B}^{n}, \varphi_{z}$ has the following properties:

(1) $\varphi_{z}(0)=z$ and $\varphi_{z}(z)=0$,

(2) $\varphi_{z}$ is an involution, i.e. $\varphi_{z} \circ \varphi_{z}=i d$, the identity mapping,

(3) the identity

$$
1-\left|\varphi_{z}(w)\right|^{2}=\frac{\left(1-|z|^{2}\right)\left(1-|w|^{2}\right)}{1-2\langle w, z\rangle+|w|^{2}|z|^{2}}
$$

holds for every $z, w \in \mathbb{B}^{n}$,

(4) the identity

$$
\left|\varphi_{z}^{\prime}(w)\right|=\frac{1-|z|^{2}}{1-2\langle w, z\rangle+|w|^{2}|z|^{2}}
$$

holds for every $z, w \in \mathbb{B}^{n}$.

Theorem 3.2. If $f \in B C\left(\mathbb{B}^{n}\right)$, the space of all bounded continuous functions on $\mathbb{B}^{n}$, then for each $z \in \mathbb{B}^{n}$,

$$
\tilde{T}_{f}^{(\alpha)}(z) \rightarrow f(z)
$$

as $\alpha \rightarrow \infty$ through the integers.

For $f \in C\left(\overline{\mathbb{B}^{n}}\right)$, the convergence is even uniform on $\mathbb{B}^{n}$.

Proof. By the definitions,

$$
\begin{aligned}
\tilde{T}_{f}^{(\alpha)}(z) & =\frac{\left\langle T_{f}^{\alpha} R_{\alpha z}, R_{\alpha z}\right\rangle}{R_{\alpha}(z, z)}=\frac{\left\langle Q_{\alpha} f R_{\alpha z}, R_{\alpha z}\right\rangle}{R_{\alpha}(z, z)}=\frac{\left\langle f R_{\alpha z}, R_{\alpha z}\right\rangle}{R_{\alpha}(z, z)} \\
& =\int_{\mathbb{B}^{n}} \frac{f(w) R_{\alpha z}(w) \frac{R_{\alpha z}(w)}{R_{\alpha}(z, z)} d A_{\alpha}(w)}{} \\
& =\int_{\mathbb{B}^{n}} f(w) \frac{\left|R_{\alpha}(z, w)\right|^{2}}{R_{\alpha}(z, z)} d A_{\alpha}(w) .
\end{aligned}
$$

Also, by the reproducing property,

$$
\int_{\mathbb{B}^{n}} f(z) \frac{\left|R_{\alpha}(z, w)\right|^{2}}{R_{\alpha}(z, z)} d A_{\alpha}(w)=f(z) \frac{\left\langle R_{\alpha z}, R_{\alpha z}\right\rangle}{R_{\alpha}(z, z)}=f(z) .
$$


Hence

$$
\begin{aligned}
\tilde{T}_{f}^{(\alpha)}(z)-f(z) & =\int_{\mathbb{B}^{n}}(f(w)-f(z)) \frac{\left|R_{\alpha}(z, w)\right|^{2}}{R_{\alpha}(z, z)} d A_{\alpha}(w) \\
& =\int_{\left|\varphi_{z}(w)\right| \leq \delta}+\int_{\left|\varphi_{z}(w)\right|>\delta}
\end{aligned}
$$

for any $0<\delta<1$.

By the continuity of $f$, we may, for each fixed $z$ and $\varepsilon>0$, choose $\delta>0$ so small that

$$
|f(w)-f(z)|<\varepsilon
$$

whenever $\left|\varphi_{z}(w)\right|<\delta$. Then

$$
\int_{\left|\varphi_{z}(w)\right| \leq \delta} \leq \varepsilon \underbrace{\int \frac{\left|R_{\alpha}(z, w)\right|^{2}}{R_{\alpha}(z, z)} d A_{\alpha}(w)}_{1}=\varepsilon .
$$

To estimate the second integral we use the following lemma:

Lemma 3.3. There exist constants $c$ and $C$, depending only on $\alpha$ and $n$, such that for all $z, w \in \mathbb{B}^{n}$,

$$
\left|R_{\alpha}(z, w)\right| \leq \frac{C}{[z, w]^{\frac{n+\alpha}{2}}}
$$

and

$$
\frac{c}{[z, z]^{\frac{n+\alpha}{2}}} \leq R_{\alpha}(z, z) \leq \frac{C}{[z, z]^{\frac{n+\alpha}{2}}} .
$$

Here, for the sake of brevity, we have introduced the notation

$$
[z, w]=1-2\langle z, w\rangle+|z|^{2}|w|^{2} .
$$

Postponing the proof of the lemma for a moment, using (9) and (10) we can estimate the integral over $\left|\varphi_{z}(w)\right| \geq \delta$ by

$$
\begin{aligned}
\int_{\left|\varphi_{z}(w)\right|>\delta} & \leq 2\|f\|_{\infty} \int_{\left|\varphi_{z}(w)\right|>\delta} \frac{\left|R_{\alpha}(z, w)\right|^{2}}{R_{\alpha}(z, z)} d A_{\alpha}(w) \\
& \leq 2\|f\|_{\infty} \frac{C^{2}}{c} \int_{\left|\varphi_{z}(w)\right|>\delta} \frac{\left(1-|z|^{2}\right)^{n+\alpha}}{[z, w]^{n+\alpha}} d A_{\alpha}(w) \\
& =2\|f\|_{\infty} \frac{C^{2}}{c} c_{\alpha} \int_{\left|\varphi_{z}(w)\right|>\delta} \frac{\left(1-|z|^{2}\right)^{n+\alpha}\left(1-|w|^{2}\right)^{n+\alpha}}{[z, w]^{n+\alpha}} \frac{d w}{\left(1-|w|^{2}\right)^{n}} \\
& =2\|f\|_{\infty} \frac{C^{2}}{c} c_{\alpha} \int_{\left|\varphi_{z}(w)\right|>\delta}\left(1-\left|\varphi_{z}(w)\right|^{2}\right)^{n+\alpha} \frac{d w}{\left(1-|w|^{2}\right)^{n}}
\end{aligned}
$$

Note that the measure

$$
d \lambda(x)=\frac{d x}{\left(1-|x|^{2}\right)^{n}}
$$

is invariant on $\mathbb{B}^{n}$, in the sense that

$$
d \lambda\left(\varphi_{a}(x)\right)=\frac{\left|\varphi_{a}^{\prime}(x)\right|^{n} d x}{\left(1-\left|\varphi_{a}(x)\right|^{2}\right)^{n}}=d \lambda(x),
$$

by Lemma 3.1 parts (3) and (4). 
Hence, making the change of variable $\varphi_{z}(w)=x$, we can continue with

$$
\begin{aligned}
\int_{\left|\varphi_{z}(w)\right|>\delta} & \leq 2\|f\|_{\infty} \frac{C^{2}}{c} c_{\alpha} \int_{|x|>\delta}\left(1-|x|^{2}\right)^{n+\alpha} d \lambda(x) \\
& =2\|f\|_{\infty} \frac{C^{2}}{c} c_{\alpha} \int_{|x|>\delta}\left(1-|x|^{2}\right)^{\alpha} d x \\
& \leq 2\|f\|_{\infty} \frac{C^{2}}{c} c_{\alpha}\left(1-\delta^{2}\right)^{\alpha} .
\end{aligned}
$$

Since $c_{\alpha} \sim \alpha^{n / 2}$ as $\alpha \rightarrow \infty$, the right-hand side tends to zero as $\alpha \rightarrow \infty$.

Hence

$$
\lim _{\alpha \rightarrow \infty}\left(\int_{\left|\varphi_{z}(w)\right| \leq \delta}+\int_{\left|\varphi_{z}(w)\right|>\delta}\right) \leq \varepsilon .
$$

If $f$ is not only bounded and continuous on the ball but even continuous on its closure, then for each $\varepsilon>0$ we can choose $\delta>0$ so that (8) holds for all $z \in \mathbb{B}^{n}$ simultaneously, by uniform continuity. This completes the proof of Theorem 3.2 .

The proof of (9) actually occurs in [8, Lemma 3.1] taking $t=0, s=\alpha$ in the operator $Q_{s, t}$ there. For $|y|=1$, see also [6, Lemma 2.7]. A simple proof of (10) can be found in [9, Proposition 4.1]. For $\alpha$ an integer, it is possible to give another proof using the formula (2.2). Since this might be useful for other applications we include it for completeness.

Proof of Lemma 3.3. Consider the sets of functions

$$
\begin{aligned}
A_{\beta, r} & :=\left\{\frac{p(t, z, w)}{[t z, w]^{\beta / 2}}\left(\frac{1-t^{2}|z|^{2}|w|^{2}}{[t z, w]^{1 / 2}}\right)^{r}: p \text { a polynomial }\right\} \\
A_{\beta} & :=\left\{\frac{p(t, z, w)}{[t z, w]^{\beta / 2}} q\left(\frac{1-t^{2}|z|^{2}|w|^{2}}{[t z, w]^{1 / 2}}\right): p, q \text { polynomials }\right\} .
\end{aligned}
$$

Differentiation yields

$$
\begin{aligned}
t \frac{d}{d t} A_{\beta, r}= & t \frac{d}{d t}\left(\frac{p(t, z, w)\left(1-t^{2}|z|^{2}|w|^{2}\right)^{r}}{[t z, w]^{\frac{\beta+r}{2}}}\right) \\
= & \frac{t \frac{\partial p}{\partial t}(t, z, w) \cdot\left(1-t^{2}|z|^{2}|w|^{2}\right)}{[t z, w]^{\frac{\beta+r}{2}}} \cdot\left(1-t^{2}|z|^{2}|w|^{2}\right)^{r-1} \\
& +\frac{t \cdot r \cdot p(t, z, w)\left(-2 t|z|^{2}|w|^{2}\right)}{[t z, w]^{\frac{\beta+r}{2}}} \cdot\left(1-t^{2}|z|^{2}|w|^{2}\right)^{r-1} \\
& -\frac{\frac{\beta+r}{2} \cdot p(t, z, w)\left(1-t^{2}|z|^{2}|w|^{2}\right)^{r} \cdot t \cdot\left(-2\langle z, w\rangle+2 t|z|^{2}|w|^{2}\right)}{[t z, w]^{\frac{\beta+r}{2}}+1} .
\end{aligned}
$$

Since

$$
\begin{aligned}
-2 t\langle z, w\rangle+2 t^{2}|z|^{2}|w|^{2} & =[t z, w]-1-t^{2}|z|^{2}|w|^{2}+2 t^{2}|z|^{2}|w|^{2} \\
& =[t z, w]-1+t^{2}|z|^{2}|w|^{2}=-\left(1-t^{2}|z|^{2}|w|^{2}\right)+[t z, w],
\end{aligned}
$$


the last term in (12) can be rewritten as

$$
\begin{aligned}
& -\frac{\frac{\beta+r}{2} \cdot p(t, z, w) \cdot\left(1-t^{2}|z|^{2}|w|^{2}\right)}{[t z, w]^{\frac{\beta+r}{2}}} \cdot\left(1-t^{2}|z|^{2}|w|^{2}\right)^{r-1} \\
& +\frac{\frac{\beta+r}{2} \cdot p(t, z, w) \cdot\left(1-t^{2}|z|^{2}|w|^{2}\right)^{r+1}}{[t z, w]^{\frac{\beta+r+2}{2}}} .
\end{aligned}
$$

Thus we get

$$
\begin{aligned}
& t \frac{d}{d t} A_{\beta, r} \in A_{\beta+1, r-1}+A_{\beta+1, r+1}, \quad r>0, \\
& t \frac{d}{d t} A_{\beta, 0} \in A_{\beta+1,0}+A_{\beta+1,1}, \quad r=0 .
\end{aligned}
$$

For arbitrary $c \in \mathbb{R}$ we thus get

$$
\left(t \frac{d}{d t}+c\right) A_{\beta, r} \in A_{\beta}+A_{\beta+1}
$$

and

$$
\mathcal{D}_{\alpha+1} A_{\beta, r} \in A_{\beta}+\cdots+A_{\beta+\alpha+1} .
$$

Applying this to $\frac{1-t^{2}|z|^{2}|w|^{2}}{[t z, w]^{n / 2}} \in A_{n-1,1}$ we thus obtain by (2.2)

$$
R_{\alpha}(z, w) \in A_{n-1}+\cdots+\left.A_{n+\alpha}\right|_{t=1} .
$$

Now

$$
\begin{aligned}
{[z, w] } & =1-2\langle z, w\rangle+|z|^{2}|w|^{2} \geq 1-2|z||w|+|z|^{2}|w|^{2} \\
& =(1-|z||w|)^{2}>0
\end{aligned}
$$

and

$$
[z, w] \leq 1+2|z||w|+|z|^{2}|w|^{2}=(1+|z||w|)^{2} \leq 2^{2}
$$

so

$$
0 \leq \frac{1-|z|^{2}|w|^{2}}{[z, w]^{1 / 2}} \leq \frac{1-|z|^{2}|w|^{2}}{1-|z||w|}=1+|z||w| \leq 2,
$$

and thus every function from $A_{\beta}$ has for $t=1$ the form

$$
\frac{\text { something bounded on } \mathbb{B}^{n}}{[z, w]^{\beta / 2}} \text {. }
$$

From (13) we thus get

$$
R_{\alpha}(z, w)=\frac{\text { something bounded on } \mathbb{B}^{n}}{[z, w]^{(n+\alpha) / 2}},
$$

which proves (9).

To prove the second half of the lemma, note that for $z=w$,

$$
\begin{aligned}
R_{\alpha}(z, z) & =\left(t \frac{d}{d t}+n\right) \ldots\left(t \frac{d}{d t}+n+\alpha\right) \frac{1-t^{2}|z|^{4}}{\left(1-t|z|^{2}\right)^{n}} \\
& =\left(t \frac{d}{d t}+n\right) \ldots\left(t \frac{d}{d t}+n+\alpha\right) \frac{1+t|z|^{2}}{\left(1-t|z|^{2}\right)^{n-1}} .
\end{aligned}
$$


Now again, for any polynomial $p$,

$$
\begin{aligned}
\left(t \frac{d}{d t}+c\right) \frac{p\left(t,|z|^{2}\right)}{\left(1-t|z|^{2}\right)^{\beta}=} & c \frac{p\left(t,|z|^{2}\right)}{\left(1-t|z|^{2}\right)^{\beta}}+\frac{t \frac{\partial}{\partial t} p\left(t,|z|^{2}\right)}{\left(1-t|z|^{2}\right)^{\beta}}+\frac{t p\left(t,|z|^{2}\right) \beta|z|^{2}}{\left(1-t|z|^{2}\right)^{\beta+1}} \\
= & \frac{\left(c p\left(t,|z|^{2}\right)+t \frac{\partial}{\partial t} p\left(t,|z|^{2}\right)\right)\left(1-t|z|^{2}\right)}{\left(1-t|z|^{2}\right)^{\beta+1}} \\
& +\frac{t p\left(t,|z|^{2}\right) \beta|z|^{2}}{\left(1-t|z|^{2}\right)^{\beta+1}} \\
= & \frac{p^{*}\left(t,|z|^{2}\right)}{\left(1-t|z|^{2}\right)^{\beta+1}},
\end{aligned}
$$

where $p^{*}$ is also a polynomial, which further satisfies $p^{*}(1,1)=\beta p(1,1)$. By iteration and taking $|z|=1, t=1$, we get

$$
R_{\alpha}(z, z)=\frac{P\left(|z|^{2}\right)}{\left(1-|z|^{2}\right)^{n+\alpha}}
$$

where $P$ is a polynomial satisfying

$$
P(1)=2 \frac{(n+\alpha-1) !}{(n-2) !} .
$$

Thus $R_{\alpha}(z, z)\left(1-|z|^{2}\right)^{n+\alpha}$ is a positive function inside $\mathbb{B}^{n}$, which has a nonzero finite limit (14) at the boundary. Consequently, it has positive and finite lower and upper bounds $c$ and $C$, respectively. This concludes the proof of Lemma 3.3 .

Corollary 3.4. For any $f \in C\left(\overline{\mathbb{B}^{n}}\right)$,

$$
\left\|T_{f}^{(\alpha)}\right\| \longrightarrow\|f\|_{\infty} \quad \text { as } \alpha \rightarrow \infty .
$$

Proof. From the Schwarz inequality we get

$$
\begin{aligned}
\left|\tilde{T}_{f}^{(\alpha)}(z)\right| & =\frac{\left|\left\langle T_{f}^{(\alpha)} R_{\alpha z}, R_{\alpha z}\right\rangle\right|}{R_{\alpha}(z, z)}=\frac{\left|\left\langle f R_{\alpha z}, R_{\alpha z}\right\rangle\right|}{R_{\alpha}(z, z)} \leq \frac{\left\|f R_{\alpha z}\right\|_{L^{2}}\left\|R_{\alpha z}\right\|_{L^{2}}}{\left\|R_{\alpha z}\right\|_{L^{2}}^{2}} \\
& \leq \frac{\|f\|_{\infty}\left\|R_{\alpha z}\right\|_{L^{2}}^{2}}{\left\|R_{\alpha z}\right\|_{L^{2}}^{2}}=\|f\|_{\infty} .
\end{aligned}
$$

Taking the supremum over all $z$ gives

$$
\left\|\tilde{T}_{f}^{(\alpha)}\right\|_{\infty} \leq\|f\|_{\infty}
$$

On the other hand, by Theorem 3.2 .

$$
\tilde{T}_{f}^{(\alpha)}(z) \longrightarrow f(z)
$$

for all $z \in \mathbb{B}^{n}$. As $\left\|\tilde{T}_{f}^{(\alpha)}\right\|_{\infty} \geq\left|\tilde{T}_{f}^{(\alpha)}(z)\right|$ and $\left|\tilde{T}_{f}^{(\alpha)}(z)\right| \rightarrow|f(z)|$, thus

$$
\liminf _{\alpha \rightarrow \infty}\left\|\tilde{T}_{f}^{(\alpha)}\right\| \geq|f(z)| .
$$

Taking again the supremum over all $z \in \mathbb{B}^{n}$ yields

$$
\liminf _{\alpha \rightarrow \infty}\left\|\tilde{T}_{f}^{(\alpha)}\right\|_{\infty} \geq \sup _{z \in \mathbb{B}^{n}}|f(z)|=\|f\|_{\infty} .
$$


From (15) and (16) we therefore have

$$
\lim _{\alpha \rightarrow \infty}\left\|\tilde{T}_{f}^{(\alpha)}\right\|=\|f\|_{\infty}
$$

which proves the "deformation estimate" (2).

\section{REFERENCES}

[1] L. V. Ahlfors: Möbius transformations in several dimensions, University of Minnesota, Minneapolis, 1981. MR725161 (84m:30028)

[2] N. Aronszajn: Theory of reproducing kernels, Trans. Amer. Math. Soc. 68, 1950, 337-404. MR0051437 (14:479c)

[3] S. Axler, P. Bourdon, W. Ramey: Harmonic Function Theory, Springer-Verlag, New York, 2001. MR1805196 (2001j:31001)

[4] M. Bordemann, E. Meinrenken, M. Schlichenmaier: Toeplitz quantization of Kähler manifolds and $\operatorname{gl}(N), N \rightarrow \infty$ limits, Comm. Math. Phys. 165, 1994, 281-296. MR1301849 (96f:58067)

[5] R. R. Coifman, R. Rochberg: Representation theorems for holomorphic and harmonic functions in $L^{p}$, Astérisque 77, 1980, 11-66. MR604369 (82j:32015)

[6] M. Jevtić, M. Pavlović: Harmonic Bergman functions on the unit ball in $\mathbb{R}^{n}$, Acta Math. Hungar. 85, 1999, 81-96. MR1713093 (2001e:31005)

[7] C. Liu: A "deformation estimate" for the Toeplitz operators on harmonic Bergman spaces, Proc. Amer. Math. Soc. 135, 2007, 2867-2876. MR2317963

[8] C. Liu, J. Shi, G. Ren: Duality for harmonic mixed-norm spaces in the unit ball of $\mathbb{R}^{n}$, Ann. Sci. Math. Québec 25, 2001, 179-197. MR1914585 (2003g:46026)

[9] J. Miao: Reproducing kernels for harmonic Bergman spaces of the unit ball, Monatsh. Math. 125, 1998, 25-35. MR1485975 (98k:46042)

Mathematical Institute, Silesian University in Opava, Na RybníčKu 1, 74601 Opava, Czech Republic

E-mail address: Renata.Otahalova@math.slu.cz 